\title{
P-ANCA in monozygotic twins with inflammatory bowel disease
}

\author{
P Yang, G Järnerot, D Danielsson, C Tysk, E Lindberg
}

\begin{abstract}
Perinuclear antineutrophil cytoplasmic antibodies (P-ANCA) have been demonstrated in patients with ulcerative colitis and in a higher frequency than expected in their first degree relatives. A hypothesis was proposed that $P$-ANCA is genetically determined and may represent a subclinical marker of genetic susceptibility to ulcerative colitis. This study analysed P-ANCA in monozygotic twins with inflammatory bowel disease to evaluate this hypothesis further. P-ANCA was analysed with indirect immunofluorescence technique in 12 monozygotic twin pairs with ulcerative colitis and 14 twin pairs with Crohn's disease. Furthermore, the study included 21 non-twin patients with ulcerative colitis, 18 non-twin patients with Crohn's disease, and 52 healthy controls matched for sex and age. In ulcerative colitis $\mathbf{P}$-ANCA occurred in nine of $14(64 \cdot 3 \%)$ monozygotic twins and in 13 of $21(61.9 \%)$ non-twin cases, which was significantly different compared with healthy controls who were positive in three of $52(5 \cdot 8 \%)$ cases $(p<0.0001)$. P-ANCA was found in two of $10(20 \%)$ healthy twin siblings to twins with ulcerative colitis, which was not significantly different from healthy controls $(p=0 \cdot 18)$. The results in Crohn's disease did not differ from healthy controls. Previous findings of P-ANCA occurring in ulcerative colitis but not in Crohn's disease are supported. This study does not support the hypothesis that P-ANCA is a subclinical marker of genetic susceptibility to ulcerative colitis. (Gut 1995; 36: 887-890)

Keywords: P-ANCA, inflammatory bowel disease, monozygotic twins.
\end{abstract}

Department of

Medicine, Division of

Gastroenterology and

Department of Clinical

Microbiology and

Immunology, Örebro

Medical Center

Hospital, Örebro,

Sweden

P Yang

G Järnerot

D Danielsson

C Tysk

E Lindberg

Correspondence to: Dr G Järnerot, Department of Medicine, Division of Gastroenterology, Orebro Medical Center Hospital,

Accepted for publication

11 October 1994

During recent years it has been firmly established that perinuclear antineutrophil cytoplasmic antibody (P-ANCA) is a frequent finding in ulcerative colitis (UC) but not in Crohn's disease. ${ }^{12}$ A North American family study showed P-ANCA in 14 of 93 (15\%) first degree relatives to $38 \mathrm{UC}$ patients in Los Angeles and in nine of $43(21 \%)$ first degree relatives to 22 UC patients in Calgary, Alberta, Canada. ${ }^{3}$ In the Los Angeles study population three of $15(20 \%)$ second degree relatives were positive. A German study of 43 UC patients and 142 first degree relatives, so far only reported in abstract form, showed $70 \%$ of the UC patients and $30 \%$ of their first degree relatives to be P-ANCA positive compared with $0 \%$ in controls. ${ }^{4}$ These findings have raised the hypothesis that this antibody may be a potential marker of genetic susceptibility to UC. In contrast, in a French study in which 15 of 30 patients with UC had P-ANCAs, none of their 91 first degree or nine second degree relatives were P-ANCA-positive. ${ }^{5}$ Neither could two recent studies confirm an increased prevalence of P-ANCA in first degree relatives to patients with UC. ${ }^{7}$ The discrepancy between the various studies could be caused by the difficulty in distinguishing between genetic and environmental factors. Twin studies could be of great help in this respect. Monozygotic twins have identical genes and share environmental factors while first degree relatives share environment but only half of the genes are common. The aim of this study was to evaluate the genetic influence on the occurrence of P-ANCA in a monozygotic twin population earlier reported from our department. ${ }^{8-11}$

\section{Methods}

Twins

Our studied twin population has earlier been described in detail. ${ }^{8-11}$ Thirty four unselected monozygotic twin pairs with inflammatory bowel disease (IBD) were found in that study. Those who were younger than 75 years of age and with both twins in each pair still alive were invited to participate in the investigation. Two recently diagnosed monozygotic twin pairs with IBD were also invited. Fifty two of 66 subjects agreed to participate constituting 14 pairs with Crohn's disease and 12 pairs with UC. Five pairs with Crohn's disease and two pairs with UC were concordant for the disease. The study was approved by the ethical committee, Örebro Medical Center Hospital.

Non-twin IBD cases
Thirty nine patients with IBD (21 UC, 18 Crohn's disease) attending our colitis clinic were tested for the occurrence of P-ANCA. Eleven of the UC patients were considered to have active disease estimated from conventional clinical and endoscopic criteria while 10 were inactive. In the Crohn's disease group five were active and 13 inactive. None of these patients were treated with corticosteroids or azathioprine at the time of the study.

Healthy controls

One healthy control subject for each twin was chosen from members of the staff or blood 
donors, all without any history of gastrointestinal disease. In total, 52 subjects were matched for sex and age within two years.

\section{$P-A N C A$}

P-ANCA was detected in ethanol fixed neutrophils by indirect immunofluorescence. ${ }^{12}$ Briefly, heparinised venous blood was drawn from two healthy members of the staff and neutrophils were separated by Ficoll-Paque (Kabi-Pharmacia, Uppsala, Sweden) density centrifugation according to the method of Böyum. ${ }^{13}$ Glass slides with neutrophils on a well defined area were prepared by means of cytocentrifugation (Shandon Cytospin, Cheshire, England), fixed in fresh $99 \%$ ethanol for 5 to 10 minutes at $+4^{\circ} \mathrm{C}$, air dried, and stored in the refrigerator until use. Serum samples from patients and healthy controls were diluted 1:10 in phosphate buffered saline, $\mathrm{pH}$ $7 \cdot 4$; and then tested for the presence of P-ANCA. Approximately $33 \mu \mathrm{l}$ of the diluted serum was applied on the well defined area of the glass slide with neutrophils and incubated in a humid chamber for 30 minutes at room temperature. After washing with phosphate buffered saline for 10 minutes and incubation for another 30 minutes with a 1:10 dilution of FITC labelled $F(a b) 2$ rabbit antihuman IgG antibodies (Dakopatts A/S, Copenhagen, Denmark), the slides were washed again in phosphate buffered saline for 10 minutes and finally mounted under a cover slip with phosphate buffered saline-glycerin. The slides were scrutinised at a magnification of $250 \mathrm{X}$ or $420 \mathrm{X}$ under a Zeiss epifluorescence microscope equipped with a $50 \mathrm{~W}$ tungsten lamp, BD $450-490$ as primary filter and LP520 as barrier filter. Serum samples giving a typical perinuclear staining reaction with the neutrophils were regarded as positive. They were always retested at the initial dilution of $1: 10$ and further at twofold dilutions of 1:20-1:640. All samples were analysed blindly, and the code was broken when all the analyses had been performed.

\section{Statistics}

Fisher's exact test and McNemar test were used for statistical analysis, and a $p$ value $<0.05$ was considered statistically significant.

\section{Results}

\section{Patients}

In twins with UC the mean age at diagnosis was 27.7 years $(17-45)$ and the actual mean age was $49 \cdot 1$ years (24-74). All were in clinical remission with normal concentrations of haemoglobin, $\mathrm{C}$ reactive protein, and serum orosomucoid. Four patients had an ileostomy. In the others the rectal mucosa was macro and microscopically inactive.

The mean age at diagnosis in twins with Crohn's disease was 28.5 years (20-45), and the actual mean age was 42.9 years (34-63). Two twins had mild diarrhoea and slightly increased $\mathrm{C}$ reactive protein and orosomucoid
TABLE I Distribution and results of $P$ - $A N C A$ in monozygotic twins concordant or discordant for ulcerative colitis

\begin{tabular}{lllll}
\hline Twin pair number & \multicolumn{2}{c}{ Distribution of disease } & \multicolumn{2}{c}{ Titre of $P$ - $A N C A$} \\
\hline 1 & UC & H & 0 & 0 \\
2 & UC & H & $1 / 80$ & 0 \\
3 & UC & H & 0 & 0 \\
4 & UC & H & $1 / 40$ & 0 \\
5 & UC & H & 0 & $1 / 640$ \\
6 & UC & UC & 0 & $1 / 20$ \\
7 & UC & H & $1 / 40$ & $1 / 40$ \\
8 & UC & H & $1 / 20$ & 0 \\
9 & UC & UC & $1 / 20$ & $1 / 160$ \\
10 & UC & H & 0 & 0 \\
11 & UC & H & $1 / 10$ & 0 \\
12 & UC & H & $1 / 10$ & 0 \\
\hline
\end{tabular}

$\mathrm{UC}=$ twin with ulcerative colitis; $\mathrm{H}=$ healthy twin sibling

concentrations. They were treated with sulphasalazine. The remaining twins were in remission. Three of them had an ileostomy.

A thorough interview did not reveal symptoms suggestive of IBD in the healthy twins. They had remained healthy for an average of 21.4 years (8-40) after diagnosis in the twins with UC and 14.9 years $(7-31)$ in the twins with Crohn's disease. All had a normal rectal mucosa and histopathological examinations were normal as well.

In the non-twin IBD group the UC patients had a mean age of $44(21-73)$ years and the Crohn's disease group 47 (20-81) years.

\section{$P-A N C A$}

Positive P-ANCA refers to a perinuclear staining pattern whereas no case of cytoplasmatic staining was found.

Nine of $14(64 \cdot 3 \%)$ identical twins with UC had a positive P-ANCA and 13 of $21(61.9 \%)$ non-twin UC cases. Of the 10 healthy monozygotic twins two had P-ANCA $(20 \%)$. In one of these two twin pairs both the diseased and the healthy twin were P-ANCA positive. In the other pair the healthy twin had a high P-ANCA titre $(1 / 640)$ while the diseased twin was negative. In the two pairs concordant for UC both twins in one pair were P-ANCA positive while in the other pair one was positive and the other P-ANCA negative (Table I)

One of $19(5 \cdot 3 \%)$ of identical twins with Crohn's disease had a positive P-ANCA and one of $18(5 \cdot 6 \%)$ non-twin CD cases. Of the nine healthy monozygotic twins none had a positive P-ANCA. Of the healthy control subjects three of $52(5.8 \%)$ had a positive P-ANCA. Table II summarises the results.

TABLE II Distribution of $P-A N C A$ in all monozygotic twins with ulcerative colitis $\left(U C_{\mathrm{tw}}\right)$ and their healthy twin siblings $\left(H-U C_{\mathrm{tw}}\right)$, in monozygotic twins with Crohn's disease $\left(C D_{\mathrm{tw}}\right)$ and their healthy twin siblings $\left(H-C D_{\mathrm{tw}}\right)$, in non-twin cases with ulcerative colitis (UC) and Crohn's disease $\left(C D_{\mathrm{c}}\right)$, and in healthy controls

\begin{tabular}{lccl}
\hline & $P-A N C A+$ & $P-$ ANCA - & Statistics $^{\star}$ \\
\hline $\mathrm{UC}_{\mathrm{tw}}$ & 9 & 5 & $\mathrm{p}<0 \cdot 0001$ \\
$\mathrm{H}_{\mathrm{tw}}$ & 2 & 8 & $\mathrm{p}=0 \cdot 18$ \\
$\mathrm{CD}_{\mathrm{tw}}$ & 1 & 18 & $\mathrm{p}=0 \cdot 71$ \\
$\mathrm{H}_{\mathrm{tw}}$ & 0 & 9 & $\mathrm{p}=0 \cdot 61$ \\
$\mathrm{UC}_{\mathrm{c}}$ & 13 & 8 & $\mathrm{p}<0 \cdot 0001$ \\
$\mathrm{CD}_{\mathrm{c}}$ & 1 & 17 & $\mathrm{p}=0 \cdot 73$ \\
Healthy controls & 3 & 49 & $\mathrm{ND}$ \\
\hline
\end{tabular}

*Fisher's exact test versus healthy controls; ND=not done. 
TABLE III Distribution of $P$ - $A N C A$ restricted to the 10 monozygotic twin pairs discordant for ulcerative colitis

\begin{tabular}{llll}
\hline & & UC twin \\
\cline { 3 - 4 } & & $P-A N C A+$ & $P-A N C A-$ \\
\hline Healthy & $P-A N C A+$ & 1 & 1 \\
UC twin & $P-A N C A-$ & 5 & 3
\end{tabular}

McNemar test (one sided) for difference in distribution of P-ANCA+, $\mathrm{p}=0 \cdot 11$.

The prevalence of P-ANCA was significantly higher both in the monozygotic UC twins and in the non-twin UC cases compared with healthy control subjects $(p<0.0001)$. The frequency of P-ANCA in the monozygotic twins with UC was significantly higher than in the corresponding healthy twin siblings $(p=0.047$; Fisher's exact test). The distribution of P-ANCA within the 10 twin pairs discordant for UC showed a difference which, however, did not reach statistical significance ( $p=0.11$; McNemar test) (Table III). Obviously, this is an effect of the limited number of cases in this analysis. In the Crohn's disease groups no statistical differences occurred between twins, non-twin cases, and healthy controls. The occurrence of P-ANCA was not influenced by disease activity (not shown).

\section{Discussion}

The aetiology of UC is unknown, probably multifactorial, and dependent on the interplay of genetical and environmental factors. Complex segregation analysis has shown the presence of a major dominant/additive gene with a penetrance of $0.20-0.26$ in about $10 \%$ of the patients with UC. ${ }^{14} 15$ This means that the genotype is normal in about $90 \%$ of adult UC patients. ${ }^{15}$ The concordance rate in the twins with UC was one of $16,{ }^{8}$ which is compatible with the results in the segregation analysis. In addition to P-ANCA, several subclinical markers have been suggested to aid genetic studies in IBD. However, studies of HLA-antigens, complement allotypes, allotypes of immunoglobulins, $\mathrm{T}$ cell receptor chains, lymphocytotoxic antibodies, antibodies to colonic epithelial cells, and intestinal permeability have not shown any consistent patterns. ${ }^{1617}$ The analysis of colonic glycoproteins in monozygotic twins showed a genetically defined change, which occurred both in healthy twins and twins with UC. ${ }^{9}$

In UC, the antigens to which P-ANCA reacts are not fully identified, but the antibody has probably no pathophysiological importance. It is generally accepted that there is no correlation between P-ANCA and the extent, duration or clinical activity of UC and the antibody generally persists after proctocolectomy. P-ANCA may rather represent a marker of an underlying immunoregulatory disturbance. An association between HLA-DR2 and P-ANCA positive UC was reported whereas an increased frequency of HLA-DR4 was seen in P-ANCA negative UC. ${ }^{18}$ The increased frequency of P-ANCA reported in healthy first degree relatives to probands with UC raised the hypothesis that P-ANCA may be genetically determined. Furthermore, the possibility of genetic heterogeneity was suggested as the prevalence of P-ANCA in relatives to P-ANCA positive probands was significantly higher than in P-ANCA negative probands. ${ }^{3}$ If genetics are of fundamental importance for the occurrence of P-ANCA, a much higher frequency of this antibody would be expected in healthy monozygotic twins than in patients with UC compared with other first degree relatives. This was not the case in our study with twins.

We confirm previous results of P-ANCA in patients with IBD. Twenty two of all 35 UC cases $(62.9 \%)$ were P-ANCA positive and only two of all 37 cases with $C D(5 \cdot 4 \%)$. The sensitivity of P-ANCA is within the limits in most previous reports. ${ }^{2-419-22}$ Our data of twin siblings, however, do not support the hypothesis that P-ANCA might be a genetic marker in UC. This conclusion is mainly based on the findings in the healthy twin siblings to twins with manifest UC. Only two of 10 healthy twin siblings $(20 \%)$ were P-ANCA positive, which was not significantly different from healthy controls $(p=0 \cdot 18$; Fisher's exact test $)$. The frequency of P-ANCA in the monozygotic twins with UC was significantly higher than in the corresponding healthy twin siblings $(p=0.047$; Fisher's exact test). The distribution of P-ANCA within the 10 twin pairs discordant for UC showed a difference which, however, did not reach statistical significance $(p=0.11 ; M c N e m a r$ test). Obviously, this is an effect of the limited number of cases in this analysis. All performed statistical tests taken together, however, give no support for a major genetic influence on the expression of P-ANCA and the results of this study do not favour P-ANCA as a marker of genetic susceptibility of UC.

The discrepancies between our findings and those by Shanahan's group and Seibold et $a l^{34}$ could be caused by the different types of relatives studied as we restricted our study to monozygotic twins. However, there might also be methodological differences. The whole cell enzyme linked immunosorbent assay (ELISA) used by Shanahan et al ${ }^{13}$ might detect antineutrophil cytoplasmic antibodies other than those responsible for P-ANCA, which is based on morphological criteria. Although the whole cell ELISA and indirect immunofluorescence


sitivity, however, of the ELISA technique has not yet been confirmed by others.

In summary, the frequency of P-ANCA was not significantly increased in healthy monozygotic twin siblings to twins with ulcerative colitis compared with healthy controls. Our data are in accordance with the French, British, and Italian studies ${ }^{5-7}$ but in contrast with the American and German findings. ${ }^{34}$ Whether ethnical factors influence the results is unknown and cannot be stated from our data.

This study was financially supported by the Örebro County Research Committee and Örebro Medical Center Research Foundation. Eva Lindberg and Curt Tysk were financially supported for IBD studies by KABI-Pharmacia, Uppsala, Sweden. This is gratefully acknowledged. 
1 Saxon A, Shanahan F, Landers C, Ganz T, Targan S. A distinct subset of anti-neutrophil cytoplasmic antibodies is associated with inflammatory bowel disease. $\mathcal{F}$ Allergy Clin Immunol 1990; 86: 202-10.

2 Duerr RH, Targan SR, Landers CJ, Sutherland LR, Shanahan F. Anti-neutrophil cytoplasmic antibodies in ulcerative colitis. Comparison with other colitides/ diarrhoeal illness. Gastroenterology 1991; 100: 1590-6.

3 Shanahan F, Duerr RH, Rotter JI, Yang H, Sutherland LR, McElree C, et al. Neutrophil autoantibodies in ulcerative colitis: familial aggregation and genetic heterogeneity. Gastroenterology 1992; 103: 456-61.

4 Seibold F, Klein R, Slametschka D, Weber P, Berg P, Gregor $M, p-A N C A$ in family members of patients with ulcerative colitis and PSC. Gastroenterology 1993; 104: A778.

5 Reumaux D, Delecourt L, Colombel JF, Noel LH, Duthilleul P, Cortot A. Anti-neutrophil cytoplasmic autoantibodies in relatives of patients with ulcerative colitis [letter]. Gastroenterology 1992; 103: 1706.

6 Lee JCW, Cambridge G, Lennard-Jones JE. Prevalence of anti-neutrophil antibodies in familial inflammatory bowel disease: specificity for ulcerative colitis in genetically related individuals. Gastroenterology 1994; 106: A718.

7 Monteleone G, Doldo P, Marasco R, Parrello T, Imeneo $M$, DeMedici A, et al. Perinuclear neutrophil autoantibodies ( $p-A N C A)$ in unaffected relative of patients with ulcerative colitis (UC). Suggestions against familial aggregation. Gut 1994; 35 (suppl 4): S31.

8 Tysk C, Lindberg E, Järnerot G, Flodérus-Myrhed B. Ulcerative colitis and Crohn's disease in an unselected population of monozygotic and dizygotic twins. A study of population of monozygotic and dizygotic twins. A study of 990-6.

9 Tysk C, Riedesel H, Lindberg E, Panzini B, Podolsky D, Järnerot G. Colonic glycoproteins in monozygotic twins with inflammatory bowel disease. Gastroenterology 1991; 100: 419-23.

10 Lindberg E, Magnusson K-E, Tysk C, Järnerot G. Antibody (IgG, IgA and $\operatorname{IgM}$ ) to baker's yeast (Saccharomyces cerevisiae), yeast mannan, gliadin, ovalbumin and betalactoglobulin in monozygotic twins with inflammatory bowel disease. Gut 1992; 33: 909-13.

11 Helgeland L, Tysk C, Järnerot G, Kett K, Lindberg E, Danielsson D, et al. IgG subclass distribution in serum and rectal mucosa of monozygotic twins with or without inflammatory bowel disease. Gut 1992; 33: 1358-64.
12 Falk RJ, Jennette JC. Anti-neutrophil cytoplasmic autoantibodies with specificity for myeloperoxidase in patients with systemic vasculitis and idiopathic necrotizing and crescentic glomerulonephritis. $N$ Engl f Med 1988; 318: 1651-7.

13 Böyum A. Separation of blood leucocytes, granulocytes, and lymphocytes. Tissue Antigens 1974; 4: 269-74.

14 Monsén U, Iselius L, Johansson C, Hellers G. Evidence for a major additive gene in ulcerative colitis. Clin Genet 1989; 36: 411-4.

15 Orholm M, Iselius L, Sørensen TIA, Munkholm P, Langholz E, Binder V. Investigation of inheritance of chronic inflammatory bowel disease by complex segregation analysis. BMF 1993; 306: 20-4.

16 Rotter JI, Shohat T, Vadheim CM. Is IBD a genetic disease? In: Rachmilewitz D, Zimmerman J, eds. Inflammatory bowel disease. Dordrecht: Kluwer Academic, 1990. matory.

17 Teahon K, Smethurst P, Levi AJ, Menzies IS, Bjarnason I. Intestinal permeability in patients with Crohn's disease and their first degree relatives. Gut 1992; 33: 320-3.

18 Yang $\mathrm{H}$, Rotter II, Toyoda H, Landers C, Tyan D, McElre $\mathrm{CK}$, et al. Ulcerative colitis: a genetically heterogeneous disorder defined by genetic (HLA Class II) and subclini$\mathrm{cal}$ (antineutrophil cytoplasmic antibodies) markers. f Clin Invest 1993; 92: 1080-4.

19 Seibold F, Weber P, Klein R, Berg PA, Wiedman KH. Clinical significance of antibodies against neutrophils in patients with inflammatory bowel disease and primary sclerosing cholangitis. Gut 1992; 33: 657-62.

20 oudkerk cholangitis. Gut 1992; 33: 657-62. Goldschmeding R, von Blomberg BME, Pena AS, et al. Goldschmeding R, von Blomberg BME, Pena AS, et al. inflammatory bowel disease are mainly associated with inflammatory bowel disease are mainly associated with
ulcerative colitis. A correlation study between perinuclear antineutrophil cytoplasmic autoantibodies and clinical parameters, medical, and surgical treatment. Gut 1993; 34: 46-50.

21 Cambridge G, Rampton DS, Stevens TRJ, McCarthy DA Kamm M, Leaker B. Anti-neutrophil antibodies in inflammatory bowel disease: prevalence and diagnostic role. Gut 1992; 33: 668-74

22 Proujansky R, Fawcett PT, Gibney KM, Treem WR Hyams JS. Examination of anti-neutrophil cytoplasmic antibodies in childhood inflammatory bowel disease. f Pediatr Gastroenterol Nutr 1993; 17: 193-7. 